\title{
CFU-S 11 activity does not localize solely with the aorta in the aorta-gonad-mesonephros region
}

\author{
Marella F. T. R. de Bruijn, Marian C. E. Peeters, Tanya Luteijn, Pim Visser, Nancy A. Speck, and Elaine Dzierzak
}

The aorta-gonad-mesonephros (AGM) re-
gion is a potent hematopoietic site in the
midgestation mouse conceptus and first
contains colony-forming units-spleen day
11 (CFU-S ${ }_{11}$ ) at embryonic day 10 (E10).
Because CFU- S $_{11}$ activity is present in the
AGM region before the onset of hemato-
poietic stem cell (HSC) activity, CFU-S
activity in the complex developing vascu-
lar and urogenital regions of the AGM was localized. From E10 onward, CFU-S 11 activity is associated with the aortic vasculature, and is found also in the urogenital ridges (UGRs). Together with data obtained from organ explant cultures, in which up to a 16-fold increase in $\mathrm{CFU}-\mathrm{S}_{11}$ activity was observed, it was determined that $\mathrm{CFU}-\mathrm{S}_{11}$ can be increased autonomously both in vascular sites and in UGRs. Furthermore, CFU-S $\mathrm{S}_{11}$ activity is present in vitelline and umbilical vessels. This, together with the presence of CFU-S $\mathrm{S}_{11}$ in the UGRs 2 days before HSC activity, suggests both temporally and spatially distinct emergent sources of CFU-S ${ }_{11}$. (Blood. 2000;96:2902-2904)

๑) 2000 by The American Society of Hematology

\section{Introduction}

During mouse embryonic development, different hematopoietic cell types are successively generated at several locations within the conceptus. At embryonic day 7.5 (E7.5), primitive erythrocytes and their direct progenitors are found in the yolk sac (YS). ${ }^{1}$ Shortly thereafter, the first definitive, in vitro clonable hematopoietic progenitors are formed in the YS and embryo body. ${ }^{2-4}$ Cells capable of in vivo hematopoietic activity are found slightly later in both the YS and the embryo: at E9 colony-forming units-spleen day 8 $\left(\mathrm{CFU}-\mathrm{S}_{8}\right)^{5}$ and neonatal repopulating cells ${ }^{6}$ and at E10 CFU-S ${ }_{11}{ }^{5}$ and adult repopulating cells. ${ }^{7}$ We previously showed that long-term adult repopulating hematopoietic stem cells (HSCs) autonomously emerge first in the intrabody aorta-gonad-mesonephros (AGM) region (more than 34 somite pair $[\mathrm{sp}]$ ). ${ }^{8}$ More recently we subdissected the AGM region into separate pieces containing either the aorta and surrounding mesenchyme or urogenital ridges (UGRs) and demonstrated that adult repopulating HSCs are first found exclusively in the area of the dorsal aorta and not in the flanking UGRs. ${ }^{9}$ B-cell progenitors in E10 AGM were also found predominantly in the aorta/mesentery subregion. ${ }^{3}$ However, sublocalization has not been performed on $\mathrm{CFU}-\mathrm{S}_{11}$, a myeloid-restricted stem cell subset that is slightly downstream from HSCs in the adult hematopoietic differentiation hierarchy.

We previously showed that $\mathrm{CFU}-\mathrm{S}_{11}$ can be detected in the AGM region slightly earlier (32-33 sp) than adult repopulating HSCs (at least $34 \mathrm{sp}$ ). ${ }^{7,8}$ Spatial and temporal mapping of CFU-S activity in the AGM should help delineate the relationship between the functionally distinct CFU-S ${ }_{11}$ and HSCs and contribute to our insight into the complex process of the onset of definitive hematopoiesis. Here we show that unlike HSCs, which are initially localized to the area of the dorsal aorta, $\mathrm{CFU}-\mathrm{S}_{11}$ are found in both the aorta and UGRs of E10 to E12 embryos. The number of CFU-S $S_{11}$ in the aorta and in UGRs increases over developmental time. Furthermore, our data demonstrate that the aorta and UGRs are capable of autonomously increasing CFU-S $\mathrm{S}_{11}$ activity in explant cultures, suggesting that $\mathrm{CFU}-\mathrm{S}_{11}$ are generated in situ either from direct precursors or from pre-existing HSCs and expand during the in vitro culture period.

\section{Study design}

\section{Embryo generation}

C57BL $/ 10 \times$ CBA F1 female mice were mated with C57BL/10 $\times$ CBA F1 or $\ln 72^{10}$ male mice to generate embryos of appropriate ages. The day of vaginal plug discovery was considered E0. At day 10, 11, or 12 of pregnancy, the females were killed by cervical dislocation. The mice were housed according to institutional guidelines, and animal procedures were carried out in compliance with the standards for Humane Care and Use of Laboratory Animals.

\section{Cell preparation}

The embryos were taken out of the uterus, and AGMs were dissected. ${ }^{11}$ Subsequently, AGMs were subdissected into the aorta with its surrounding mesenchyme and the UGRs using 27-gauge insulin syringes (Becton Dickinson, San Jose, CA). The tissues were either cultured in 3-day explant cultures $^{8}$ or directly processed for transplantation into irradiated adult mice. Cell suspensions of freshly isolated or cultured tissues were obtained by
From the Department of Cell Biology and Genetics and the Department of Clinical Genetics, Erasmus University, Rotterdam, The Netherlands, and the Department of Biochemistry, Dartmouth Medical School, Hanover, NH.

Submitted March 21, 2000; accepted June 12, 2000.

Supported by grant 901-09-090 (M.dB. and E.D.) from the The Netherlands Organization for Scientific Research (NWO), The Hague, The Netherlands; grant EUR 99-1965 (M.P. and E.D.) from the Dutch Cancer Society, Amsterdam, The Netherlands; the Leukemia Society of America (New York, NY) award no. 103-94 (E.D.); the Fogarty Award no. FO6TW03300 (N.S.); and grants ROCA58343 (N.S.) and R01 DK51077-01 (E.D.) from the National Institutes of Health, Bethesda, MD.

Reprints: Elaine Dzierzak, Department of Cell Biology and Genetics, Erasmus University, PO Box 1738, 3000 DR Rotterdam, The Netherlands; e-mail: dzierzak@ch1.fgg.eur.nl.

The publication costs of this article were defrayed in part by page charge payment. Therefore, and solely to indicate this fact, this article is hereby marked "advertisement" in accordance with 18 U.S.C. section 1734.

(C) 2000 by The American Society of Hematology 


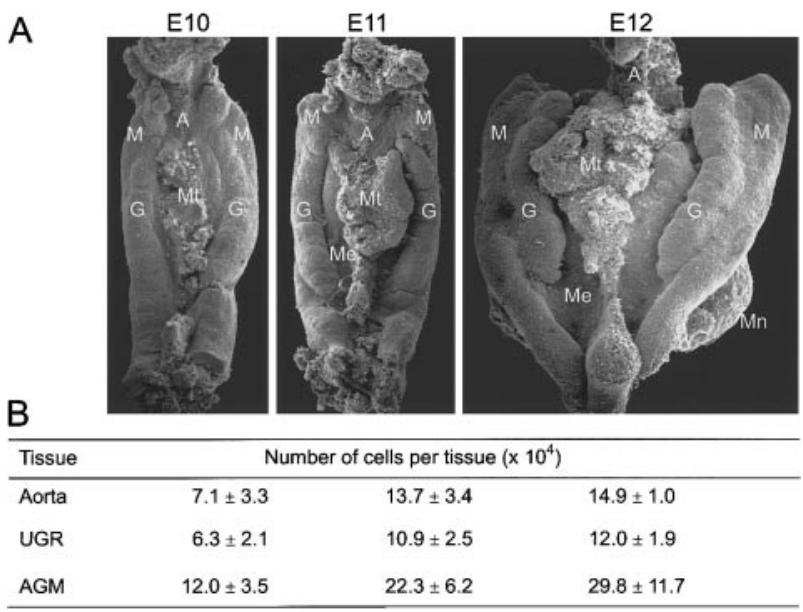

Figure 1. Changes in morphology and cell counts of the E10, E11, and E12 AGM region. (A) Scanning electron micrographs of the E10 (left), E11 (middle), and E12 (right) AGM region in which the excretory and reproductive systems are undergoing rapid growth and morphogenic changes. A indicates aorta; $G$, genital ridge/gonad; $M$, pro/mesonephros; Mn, metanephros; Me, mesenchyme; and Mt, mesentery (original magnification $\times 40$ ). (B) The ongoing organogenesis results in an increase in the cell number within the AGM from E10 to E12. Viable cell numbers for the subdissected aorta, UGRs, and entire AGM after collagenase treatment are given for each embryonic day (the mean $\pm \mathrm{SD}$ ). UGRs comprised pro/mesonephroi and genita ridges/gonads. Cell counts of E12 UGRs were obtained from counts of whole UGRs $(n=2)$ and in part from counts of UGRs further subdissected into pro/mesonephro and gonads $(n=3)$. At E12 the emerging metanephroi were not included in the cell counts of the total AGM region or UGRs. After 3 days of organ explant culture, the cell counts (given as $\times 10^{4}$ cells) for the different tissues were (1) E10: aorta, 5.6; UGRs 7.1; and AGM, 13.0; (2) E11: aorta, $5.4 \pm 2.5$; UGRs, $8.2 \pm 1.9$; and AGM $22.0 \pm 2.7$; and (3) E12: aorta, 6.0; UGRs, 18.0; and AGM, 30.8.

collagenase digestion as previously described. ${ }^{11}$ Viable cells were counted using trypan blue (Sigma Chemical, St Louis, MO).

\section{CFU-S ${ }_{11}$ assay}

Cell suspensions of AGM, aorta, or UGRs were injected intravenously into the tail vein of adult (8-14 weeks old) C57BL/10 $\times$ CBA F1 mice (male or female). The recipients were conditioned by 1000 rads of $\gamma$-irradiation, which was administered in a split dose, with 3 hours between doses. The

Figure 2. Distribution of CFU- $S_{11}$ in the aorta and UGRs. (A, B) The aorta with surrounding mesenchyme and the UGRs were dissected from E10 to E12 embryos, and cell suspensions of these tissues were directly injected into irradiated adult mice to assay for CFU- $S_{11}$. (C) Organ explant cultures (3 days) of the subdissected tissues were performed prior to transplantation. The total number of $\mathrm{CFU}_{-} \mathrm{S}_{11}$ per tissue is given in panel $\mathrm{A}$, the frequency in panel $\mathrm{B}$, and the total number after organ explant culture in panel C (fold increase compared with uncultured tissues are shown in parentheses). The data are the mean of results obtained from individually transplanted mice in a total of 14 independent experiments. CFU-S ${ }_{11}$ values (given as times $10^{4}$ cells) for the aorta and UGRs ranged from (A) E10: aorta, 0-0.3, and UGRs, 0-0.2; E11: aorta, 0-0.7, and UGRs, 0-0.35; E12: aorta, 0.42-1.0, and UGRs, 0.42-0.75; (B) E10: aorta, 0-4.2, and UGRs, 0-3.17; E11: aorta, 0-5.1, and UGRs, 0-3.2; E12: aorta, 2.8-6.6, and UGRs, 3.2-7.2; and (C) E10: aorta, 0.2-0.71, and UGRs, 0-0.27; E11: aorta, 0.22-3.14, and UGRs, 1.5-3.33; and E12, aorta 0.67-1.1, and UGRs, $0.6-0.9$. In panels $A$ and $C$, the number of $C F U-S_{11}$ per UGRs at $E 12$ was calculated from the number of CFU-S $S_{11}$ obtained from whole UGRs and partly from the number obtained from isolated gonads and pro/mesonephroi. The number of CFU-S ${ }_{11}$ obtained per embryo equivalent (ee) of whole E12 UGRs was 0.6 in panel $A$ and $0.75 \mathrm{in}$ panel $\mathrm{C}$; the number obtained from gonads and pro/mesonephroi was 0.1 and 0.4, respectively, in panel $A$ and 0 and 0.7 , respectively, in panel $C$. The frequency of $C F U-S_{11}$ (panel B) was calculated from the number of colonies per ee and the cell counts per tissue. For direct transplantations (panels $A$ and $B$ ) the following total numbers of tissues were injected: E10: both aorta and UGRs, 40 ee; E11: aorta, 53 ee, and UGRs, 47.5 ee; and E12: aorta, 22 ee; UGRs, 8 ee; gonads, 14 ee; and mesonephroi, 14 ee. For the cultured data (panel C): E10, aorta, 26 ee, and UGRs, 27 ee; E11: both aorta and UGRs, 22 ee; and E12: aorta, 26 ee; UGRs, 20 ee; gonad and mesonephri, 6 ee. (D) A summary of the distribution of HSCs in the E10 to E12 aorta and UGRs is given (from de Bruijn et al ${ }^{9}$ ). ND indicates not determined. mice were killed 11 days after transplantation by cervical dislocation. The spleens were removed and fixed in Tellesniczky's fixative, and the colonies were counted by macroscopic observation.

\section{Scanning electron microscopy}

Isolated AGMs were fixed in $2.5 \%$ glutaraldehyde (Sigma), post-fixed in $1 \%$ osmium tetroxide $\left(\mathrm{OsO}_{4}\right)$, dehydrated in ethanol, and critically pointdried. The tissues were mounted on stubs, coated with gold/paladium, and examined with a JEOL (Tokyo, Japan) JSM-25 electron microscope.

\section{Results and discussion}

The spatial and temporal distribution of CFU-S ${ }_{11}$ in the E10 to E12 AGM region was examined after subdissecting this tissue into parts containing the aorta with its surrounding mesenchyme and the UGRs. As shown in scanning electron micrographs, the AGM doubles in size from E10 to E12 (Figure 1A). During this time the

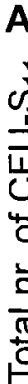

B
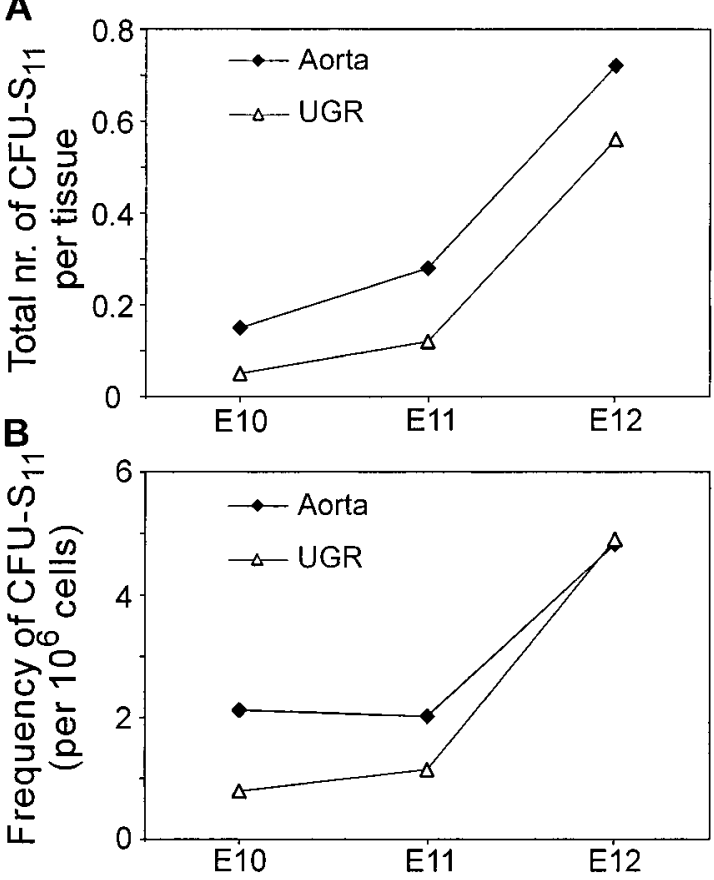

C

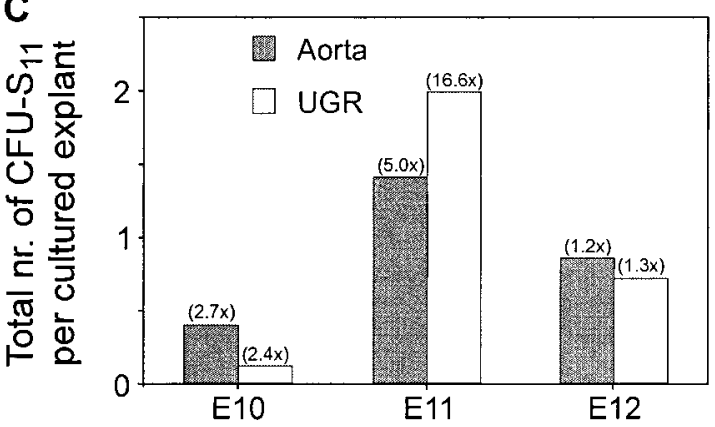

D

\begin{tabular}{|c|c|c|c|c|c|c|}
\hline \multirow{2}{*}{$\begin{array}{l}\text { HSCs } \\
\text { present }\end{array}$} & \multicolumn{2}{|c|}{ Aorta UGR } & \multicolumn{2}{|c|}{ Aorta UGR } & \multicolumn{2}{|c|}{ Aorta UGR } \\
\hline & ND & ND & ++ & - & ++ & + \\
\hline & & 10 & & & & \\
\hline
\end{tabular}

Embryonic Day 
distinctive features of the maturing excretory and reproductive systems allow for the precise separation of the UGRs from the dorsal aorta and mesenchyme. Cell counts were performed on the whole AGM, subdissected aorta, and UGRs. All tissues showed a 2-fold increase in cell number from E10 to E12 (Figure 1B). After E12, following further organogenesis, the AGM region as such recedes, and hematopoiesis takes place in the fetal liver. ${ }^{12}$

CFU-S ${ }_{11}$ were present in both the area of the aorta and UGRs at E10, E11, and E12 (Figure 2A). This was in agreement with the sublocalization of $\mathrm{CFU}-\mathrm{S}_{8}$ that was found at E10. ${ }^{13}$ A significant increase in CFU-S $\mathrm{S}_{11}$ was observed between E10 and E12, ie, 4.8-fold for the aorta and 11.2-fold for the UGRs $(P \leq .003$, as determined by linear regression analysis). At all time points there were more CFU-S $\mathrm{S}_{11}$ in the aorta than in the UGRs $(75 \%, 70 \%$, and $56 \%$ of the recovered $\mathrm{CFU}-\mathrm{S}_{11}$ for $\mathrm{E} 10, \mathrm{E} 11$, and $\mathrm{E} 12$, respectively). Also, the frequency of CFU-S $S_{11}$ was highest in the aorta at E10 and E11 (Figure 2B). Although the aorta appears to harbor more CFU-S ${ }_{11}$ than the UGRs, the precise site of CFU-S $\mathrm{S}_{11}$ origin remains uncertain. Similar to adult repopulating $\mathrm{HSCs},{ }^{9}$ CFU-S 11 may find their origin in association with the intra-embryonic vasculature. To examine this possibility, we also transplanted cells of the vitelline and umbilical vessels. At E10.5 and E11, we found 0.3 and 1.6 CFU-S ${ }_{11}$, respectively, per embryo equivalent of transplanted vitelline and umbilical cells, suggesting that CFU-S ${ }_{11}$ may be generated and/or expanded in association with vascular tissue.

The temporal increase in CFU-S $\mathrm{S}_{11}$ activity in the aorta and also in the UGRs may reflect either the autonomous generation and/or expansion of these cells in the aorta and UGRs or the influx of CFU-S ${ }_{11}$ into these tissues. To examine these possibilities, organ explants of the E10 to E12 aorta and UGRs were cultured prior to assaying CFU-S $\mathrm{S}_{11}$ activity. At E10, CFU-S $\mathrm{S}_{11}$ activity was maintained during culture (Figure 2C) and at best showed an average 2.5-fold increase compared to directly transplanted cells. In contrast, CFU-S $\mathrm{S}_{11}$ activity significantly increased in cultures of the E11 aorta (5-fold) and UGRs (16.6-fold) $(P<.05$ and $P<.005$, respectively, as determined by the Mann-Whitney U test). At E12, CFU-S activity was maintained in these cultured tissues. These data indicate that both the E11 aorta and UGRs are capable of significantly increasing CFU-S $\mathrm{S}_{11}$ in a manner that is independent of influx from other embryonic sites. This suggests that the increased CFU-S ${ }_{11}$ activity between E11 and E12 $(P<.02$, as determined by the $t$ test) reflects in situ generation and/or expansion of CFU-S $\mathrm{S}_{11}$. Interestingly, the culture of E11 UGR explants results in the greatest increase in CFU-S $\mathrm{S}_{11}$ numbers. This result is reminiscent of the dramatic increase in HSCs in the E11 UGR explant cultures we recently observed ${ }^{9}$ and leads us to speculate that this increase in CFU-S ${ }_{11}$ results from their differentiation from newly formed HSCs in the UGR cultures. Indeed, CFU-S $S_{11}$ were found in all the same sites where the first HSCs were found. However, the observation that CFU- $\mathrm{S}_{11}$ were detected in the UGRs before HSCs emerged in this site (Figure 2D) leaves open the question of how these 2 hematopoietic cell types are related to each other during early embryogenesis. A possibility is that CFU-S ${ }_{11}$ and HSCs are derived separately from different precursors, such as putative hemangioblasts. This would be in agreement with data showing multiple sites of hematopoietic birth in avian embryos. ${ }^{14}$ Thus, the CFU-S we detected may be derived by 2 different means.

In the present study we report the temporal and spatial emergence of CFU- $\mathrm{S}_{11}$ activity within the mouse AGM. We show that from mid-E10 onward, CFU-S $S_{11}$ are increasingly found both in the area of the aorta and in the UGRs, and that CFU-S $\mathrm{S}_{11}$ can be increased autonomously in both sites. It is of great interest to further examine the cells and factors involved in the increase of CFU-S $\mathrm{S}_{11}$ activity as well as the possible lineage relationships between putative hemangioblasts, CFU-S ${ }_{11}$ and HSCs. Such studies are likely to provide additional insight in the origin and ex vivo manipulation of the clinically important HSCs.

\section{Acknowledgments}

We would like to thank Alexander Medvinsky and all the members of the laboratory for their helpful discussions. We also thank Rob Ploemacher and Sjaak Philipsen for critical comments on this manuscript and Jeroen Essers for help with the figures.

\section{References}

1. Russell ES, Bernstein SE. Blood and blood formation. In: Green EL, ed. Biology of the Laboratory Mouse. New York, McGraw-Hill; 1966:351372.

2. Palis J, Robertson S, Kennedy M, Wall C, Keller G. Development of erythroid and myeloid progenitors in the yolk sac and embryo proper of the mouse. Development. 1999;126:5073-5084.

3. Godin I, Garcia-Porrero JA, Dieterlen-Lievre F, Cumano A. Stem cell emergence and hemopoietic activity are incompatible in mouse intraembryonic sites. J Exp Med. 1999;190:43-52.

4. Cumano A, Dieterlen-Lievre F, Godin I. Lymphoid potential, probed before circulation in mouse, is restricted to caudal intraembryonic splanchnopleura. Cell. 1996;86:907-916.

5. Medvinsky AL, Samoylina NL, Muller AM, Dzierzak EA. An early pre-liver intraembryonic source of CFU-S in the developing mouse. Nature. 1993; 364:64-67.

6. Yoder MC, Hiatt K, Dutt P, Mukherjee P, Bodine DM, Orlic D. Characterization of definitive lymphohematopoietic stem cells in the day 9 murine yolk sac. Immunity. 1997;7:335-344

7. Muller AM, Medvinsky A, Strouboulis J, Grosveld F, Dzierzak E. Development of hematopoietic stem cell activity in the mouse embryo. Immunity. 1994;1:291-301.

8. Medvinsky A, Dzierzak E. Definitive hematopoiesis is autonomously initiated by the AGM region. Cell. 1996;86:897-906.

9. de Bruijn MFTR, Speck NA, Peeters MCE, Dzierzak E. Definitive hematopoietic stem cells first develop within the major arterial regions of the mouse embryo. EMBO J. 2000;19:2465-2474.

10. Strouboulis J, Dillon N, Grosveld F. Developmen- tal regulation of a complete $70-\mathrm{kb}$ human betaglobin locus in transgenic mice. Genes Dev. 1992;6:1857-1864.

11. Dzierzak E, de Bruijn M. Isolation and analysis of hematopoietic stem cells of mouse embryos. In: Klug CA, Jordan CT, eds. Methods in Molecular Medicine: Hematopoietic Stem Cell Protocols. Totowa, NJ: The Humana Press, Inc. In press.

12. Dzierzak E, Medvinsky A, de Bruijn M. Qualitative and quantitative aspects of haematopoietic cell development in the mammalian embryo. Immunol Today. 1998;19:228-236.

13. Medvinsky AL, Gan OI, Semenova ML, Samoylina NL. Development of day-8 colonyforming unit-spleen hematopoietic progenitors during early murine embryogenesis: spatial and temporal mapping. Blood. 1996;87:557-566.

14. Dieterlen-Lievre F. Hematopoiesis: progenitors and their genetic program. Curr Biol. 1998;8: R727-R730. 